\title{
COMPUTERIZED DRUG THERAPY: APPLICATION OF THE HAND-HELD MICROCOMPUTER TO DOSAGE.REGIMEN DESIGN
}

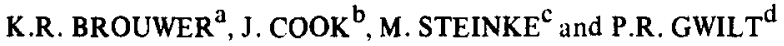 \\ ${ }^{a}$ College of Pharmacy, University' of Kentucky. ' College of Pharmacy, University of Michigan. \\ ${ }^{c}$ Department of Pharmacy, Borgess Hospital, Kalamazoo, MI and ${ }^{\mathrm{d}}$ School of Pharmacy, West \\ Virginia University, Morgantown, WV 26506 (U.S.A.)
}

(Received November 4th, 1984)

For many drugs estimation of a safe and effective dosage regimen is difficult. Typically these drugs exhibit optimal therapeutic activity when drug concentrations in the blood are maintained within narrow limits and considerable intersubject variability exists in their rate of elimination from the body.

Computer programs have been written to estimate the size and frequency of dose administration necessary to achieve therapeutic drug concentrations in the blood. The programs utilize pharmacokinetic equations and information on the individual patient's physiologic (e.g., age, weight, sex) and pathologic (e.g., existence of liver or renal disease) status.

The drugs studied were gentamicin, theophylline, digoxin, phenytoin and warfarin. The programs were written using the hand-held TRS 80 Pocket Computer with an $8 \mathrm{~K}$ memory module. These programs have been in use for 3 years and are currently involved in clinical consults approx. 100 times a month.

\section{Introduction}

It is now widely recognized that the fluctuations in serum drug concentrations during chronic dosing of medication can be modelled mathematically. Many drugs have a narrow therapeutic range i.e., the serum concentration at which the drug begins to exert its pharmacologic effect is close to the concentration at which toxicity occurs. Mathematical models can be used to determine the size and frequency of drug doses which produce serum drug concentrations within the therapeutic range thereby optimizing drug therapy.

The practical application of mathematical modelling to rational dosage regimen design has been enhanced by the availability of hand-held programmable calculators. Calculations which are time consuming and mathematically complex can now be performed at the bedside. Drugs for which calculator programs have been written to determine therapeutic doses include digoxin (Manion, 1978), phenytoin (King and Kaul, 1980), the aminoglycosides (Ng, 1980; Loertsher, 1982) and warfarin (Canaday and Sawyer, 1982).

The recent introduction of hand-held microcomputers presents a further develop- 
ment in the application of calculator/computer hardware to the design of drug therapy: Hand-held computers offer many advantages over programmable calculators of comparable price. These advantages include greater program storage, better display com. munication, easier programming, user defined functions and portability.

The purpose of this paper is to describe several programs which have been developed to adjust the size and frequency of administration of drug doses so as to achieve therapeutic concentrations. The programs have been written for the hand-held or pocket computer and incorporate algorithms currently being used clinically to establish therapeutic drug dosage regimens.

\section{Methods}

Programs were written for 5 drugs, gentamicin, theophylline, digoxin, phenytoin and warfarin. These drugs were chosen because they are used frequently, have a narrow therapeutic range and exhibit wide intersubject variability in their disposition. For these drugs a standard dose will frequently fail to produce therapeutic serum concentrations but will cause toxicity in a significant number of patients and subtherapeutic concentrations in others.

All of the programs were developed on a TRS 80 Pocket Computer and were written in BASIC. Initially the Model 1 was used but programs were updated when the PC2 was introduced. The entire set of programs can be permanently stored on the PC2 with an $8 \mathrm{~K}$ memory module.

The programs have been in use for about 3 years at Borgess Hospital, a 479 bed institution in Kalamazoo, Michigan. Consultations requiring their use average at present 80-100 consults a month, with most of these calling for the aminoglycoside program. Consults are initiated by a physician and relevant laboratory data e.g., serum drug concentrations, serum creatinine levels etc. are forwarded by the clinical laboratories. Dosage adjustment and recommendations are made by a pharmacist with training in clinical pharmacokinetics and a written report is returned to the requesting physician.

\section{Programs}

The initial portion of the program generates prompt messages asking the user which drug dosing program is required. Upon entering the specific drug dosing program, standard patient information, i.e., sex, age, height and weight is requested.

\section{Gentamicin}

This program calculates loading and maintenance infusions to maintain therapeutic gentamicin serum concentrations. The program can however be used to dose any of the aminoglycosides. Two separate algorithms are used. One estimates the loading and maintenance infusions of gentamicin on the basis of body weight and creatinine clearance alone (Hull and Sarubbi, 1978). This method is most suitable for individuals who 
are not at risk and for older patients. In younger patients and those with life threatening infections, this regimen may lead to subtherapeutic serum concentrations (Schentag, 1980). In this case the second method (Sawchuk and Zaske, 1976) which utilizes serum gentamicin concentrations obtained following an initial dose is preferred.

The first method, devised by Hull and Sarubbi (1978), requires lean body weight (LBW). This is calculated by the method of Devine (1974). Doses are then based upon LBW or total body weight if the latter is less than LBW. The patient's creatinine clearance is calculated from serum creatinine by the method of Jelliffe. The loading dose is estimated from a table relating the loading dose to the desired peak serum concentration and the patient's weight. This table has been reduced to a single empirical equation in the program.

Similarly a second table presents maintenance doses (as fractions of the loading dose) and dosing frequency based upon creatinine clearance. These relationships have also been expressed as mathematical equations. Upon completion of the program the loading dose and the frequency and size of the maintenance infusions are then printed.

For younger and high risk patients, the second algorithm for gentamicin is used. This method requires at least two gentamicin serum concentrations following an initial infusion. If the patient has been receiving the antibiotic prior to this initial infusion. a gentamicin concentration obtained immediately before the infusion is also required. Based upon these serum concentrations, the patient's elimination rate constant and volume of distribution are estimated. These parameters in turn are used to predict the size of the maintenance dose and dosing interval. A section is also included in the program allowing the user to enter an alternative dosage regimen if the previously estimated values for the dose size and frequency are not satisfactory. This section will estimate the steady state peak and trough values produced by the new regimen.

\section{Theophylline}

Using average population values of clearance and volume of distribution plus certain physiologic and pathologic characteristics of the patient, this program can be used to calculate loading and maintenance theophylline doses. If an intravenous infusion of aminophylline is indicated, an appropriate infusion rate of aminophylline is estimated to achieve a predetermined steady state theophylline serum concentration.

After the standard patient information has been entered, the desired steady state theophylline serum concentration is requested. LBW is then determined again using the equation of Devine. These equations, designed for adults, underestimate LBW for individuals with heights $36-41$ inches (roughly corresponding to ages $2-6$ ) and therefore underestimate the theophylline dose. Since many asthmatic children require theophylline an equation which better predicts LBW was used in these patients (Howard, 1980).

The volume of distribution and clearance is then selected based on the age of the patient (Hendeles et al., 1978; Ellis et al., 1976; Loughnan et al., 1976; Giacoia et al., 1976) and further adjusted to accommodate changes in theophylline disposition due 
to chronic obstructive pulmonary disease, congestive heart failure, pulmonary edema, liver disease or smoking (Powell, 1980).

The user then has the option of administering the drug intravenously or orally. For intravenous administration, a loading dose and infusion rate of aminophylline are calculated to yield the desired average steady-state plasma concentration of theophyllins. When the oral route of administration is selected, a dosing interval must also be entered. The theophylline loading dose is calculated based on total body weight, whereas the maintenance dose is determined using LBW (Schentag, 1978).

Should the estimated oral or intravenous dosage regimen appear unsatisfactory, a section of the program is written to permit the user to modify the doses and frequency of administration. Steady state peak and trough serum concentrations arising from the new regimen are then displayed.

\section{Phenytoin}

This program will calculate a therapeutic phenytoin dosage regimen based either on a single dosing rate and corresponding average phenytoin steady state plasma concentration (Winter, 1980) or on two of these values. In the latter case, the approach of Ludden et al. (1977) is used.

In patients with reduced phenytoin binding due to either low circulating albumin or the presence of uremia, a heightened response from phenytoin may be expected despite the fact that the total (bound plus unbound) concentrations are reduced. Therefore in these patients it is not feasible to adjust the dose based upon the total plasma concentration of the drug. However, by correcting for the change in albumin levels and for the presence of uremia, the phenytoin concentration can be converted to an adjusted concentration. This adjusted concentration is the concentration of total phenytoin in a patient with normal binding that would elicit the same response as observed in the patient with reduced binding. Phenytoin dosing should then be based upon this adjusted concentration (Winter, 1980).

The Michaelis-Menten equation is used to predict the therapeutic phenytoin dosage regimen.

$$
R=\frac{V_{\max } C_{\mathrm{ss}}}{K_{\mathrm{m}}+C_{\mathrm{ss}}}
$$

where $R$ is the dosing rate, usually dose per day, $C_{\mathrm{ss}}$ the steady state plasma concentration arising from that dosing rate and $V_{\max }$ and $K_{\mathrm{m}}$ are the maximum elimination rate of phenytoin and the Michaelis constant.

Once the patient characteristics have been entered, the number of steady state plasma concentrations available for dose adjustment is requested. If only one concentration is available, a population average of $4 \mu \mathrm{g} / \mathrm{ml}$ for $K_{\mathrm{m}}$ is inserted into Eqn. (1) which is then solved for $V_{\max }$. The desired therapeutic steady state concentration along with the newly derived $V_{\max }$ and the population value of $K_{\mathrm{m}}$ are re-entered into the equation and a therapeutic daily dose is computed. 
When two steady state concentrations are available, the Ludden method is used. The daily dose is plotted against the daily dose divided by the steady state plasma concentration. The slope provides $K_{\mathrm{m}}$ and the intercept on the ordinate, $V_{\max }$. The computer estimates these parameters by linear regression.

A section is included in the program to account for abnormally low serum albumin and the presence of uremia. Both of these situations would render unusually high unbound fractions of phenytoin in the plasma. This section is entered regardless of the method used to compute the therapeutic phenytoin dose. If either or both of these conditions arise, an adjusted plasma concentration is displayed. This concentration is equal to the concentration of total phenytoin in a patient with normal binding that would elicit the same pharmacologic response observed in the patient with reduced binding. Dosing should then be recalculated based upon this adjusted plasna concentration.

An opportunity is then given to the user to adjust the dosing schedule. The steady state plasma concentrations which arise from this revised schedule are displayed.

\section{Digoxin}

This program calculates a digoxin loading and maintenance dose for patients with normal or impaired renal function.

After the patient's characteristics have been entered, the route of administration (intravenous or oral) is chosen and assigned a value for the fraction of the dose absorbed. The lean body weight of the patient is determined and Jelliffe's equation is used to estimate creatinine clearance from the patient's sex, age and serum creatinine (Jelliffe, 1973). Digoxin clearance and volume of distribution are calculated using empirical equations relating these parameters to creatinine clearance (Winter, 1980). Two equations are available for digoxin clearance depending on whether the patient has congestive heart failure or not. The clearance and volume terms are corrected for body weight and used to estimate loading and daily maintenance doses necessary to achieve a selected steady state plasma digoxin concentration.

Patients often present who are already on digoxin therapy but their dosage regimen has resulted in either too low or too high digoxin plasma concentrations. A section of the program has been written which allows the user to convert these inappropriate dosing schedules to therapeutic regimens. For the patient with a low, but measurable digoxin concentration, an attenuated loading dose is calculated on the basis of the existing digoxin concentration and the desired concentration. For the patient with toxic concentrations, a computation is made of how long digoxin therapy should bc withheld before plasma concentrations return to the desired steady state level and the new maintenance schedule can be started.

Finally as in the other programs a section is included enabling the user to choose an alternative dosage regimen if the calculated schedule appears unsatisfactory. Steady state digoxin plasma concentrations arising from the new regimen are then displayed.

\section{Warfarin}

This program predicts a daily maintenance warfarin dose using the method of 
Williams and Karl (Williams and Karl, 1979). Baseline prothrombin time is measured immediately prior to anticoagulation. Loading doses of warfarin are administered daily and prothrombin times are also monitored each day. Generally, doses are administered until a prothrombin time of about $20 \mathrm{~s}$ is achieved. Following the method of Williams and $\mathrm{Karl}$, the daily prothrombin time is plotted aganst the cumulative amount of warfarin administered. The area under this curve is estimated between the prothrombin times of the baseline value and $20 \mathrm{~s}$. In a retrospective study of 105 patients a linear relationship was observed between the maintenance dose which successfully sustains a prothrombin time of $20 \mathrm{~s}$ and the area just calculated. An equation relating these two factors was developed by least squares regression. When the area was entered into the equation, an appropriate maintenance dose could be predicted.

The program requires entry of the daily warfarin doses and the corresponding prothrombin times during the loading phase. The area between the baseline and 20 -s prothrombin time is estimated using the trapezoidal rule. The area is inserted into the equation developed by Williams and Karl and the maintenance dose is displayed.

\section{Results}

These programs have been used successfully at several institutions, although most frequently at Borgess Hospital, Kalamazoo, Michigan. The following examples illustrate the use of the programs with a hand-held microcomputer in the clinical setting.

\section{Digoxin}

M.F., a 54-year-old white female, presented to the E.R. on 10/29 with increasing edema of the lower extremities, face and periorbital area and a weight gain of $5.0 \mathrm{~kg}$ over the past 3 weeks. Admission weight was $56 \mathrm{~kg}$ and her height was $160 \mathrm{~cm}$. Medications on admission included Lanoxin $0.25 \mathrm{mg}$ q.d., along with Apresoline, Lasix and Isordil. A serum creatinine on admission was reported at $1.3 \mathrm{mg} \%$. On 10/31 digoxin levels were ordered. Using the hand-held microcomputer, a digoxin level of $2.0 \mathrm{ng} / \mathrm{ml}$ was predicted, later the lab reported a level of $2.2 \mathrm{ng} / \mathrm{ml}$ and on this basis the dosing was changed to $0.125 \mathrm{mg}$ q.o.d. and $0.25 \mathrm{mg}$ q.o.d.

\section{Aminoglycosides}

W.M., a 46-year-old white female, with metastic breast cancer, was admitted to the E.R. with chest pain on $8 / 28$. She was later found to have vertebral body metastasis and was treated with radiation therapy, which was to be completed on 11/3. On 10/31 the patient developed a temperature of $102^{\circ} \mathrm{C}$ and the blood showed gram negative rods which were reported to be sensitive to Tobramyein. On 10/31 the patient was given a loading dose of $90 \mathrm{mg}$ IV Tobramycin followed $8 \mathrm{~h}$ later by $60 \mathrm{mg}$ infused over $0.5 \mathrm{~h}$ every $8 \mathrm{~h}$ at 09:00, 17:00 and 01:00 h. On 11/2 blood levels drawn at 08:30 and $10: 10 \mathrm{~h}$ revealed a trough of $2.3 \mu \mathrm{g} / \mathrm{ml}$ and a peak of $4.5 \mu \mathrm{g} / \mathrm{ml}$. The dose of Tobramycin was then changed to $50 \mathrm{mg}$ IV q12 h infused over $0.5 \mathrm{~h}$. Using the method of 
aminoglycoside dosing developed by Sawchuk and Zaske, a peak concentration of $3.7 \mu \mathrm{g} / \mathrm{ml}$ and a trough concentration of $1.1 \mu \mathrm{g} / \mathrm{ml}$ were predicted. Blood levels drawn on $11 / 4$ reported a peak concentration $3.9 \mu \mathrm{g} / \mathrm{ml}$ at $10: 00 \mathrm{~h}$ and a trough concentration of $1.4 \mu \mathrm{g} / \mathrm{ml}$ at $08: 30 \mathrm{~h}$.

\section{Warfarin}

H.C., a 57-year-old white male, status post permanent pacemaker in 1972, was admitted with a throbbing pain in his left lower leg. There was swelling from the knee down and it was hot to the touch; a venogram showed a deep vein occlusion of the left leg to the mid-thigh. Admission PT was $11.0 \mathrm{~s}$ and SMA's were within normal limits. Admission medications included Lanoxin $0.25 \mathrm{mg}$ q.d., Inderal $10 \mathrm{mg}$ t.i.d. and Cardioquin one tablet q $8 \mathrm{~h}$. The patient was started on IV Heparin 24000 units over $24 \mathrm{~h}$ which was increased to 36000 units the next day. After this the heparin was tapered and the patient was started on Coumadin according to the protocol developed by Williams and Karl. After 3 days of therapy, the PT's were plotted against the cumulative Coumadin dose and the method of Williams and Karl was used to calculate a maintenance dose. The maintenance dose indicated by this method was $5.7 \mathrm{mg} / \mathrm{day}$. The patient was then given $5.0 \mathrm{mg}$ of Coumadin/day and PT's reported at 3 and 4 days later were $20.8 \mathrm{~s}$ and $21.3 \mathrm{~s}$, respectively.

\section{References}

Canaday, B.R. and Sawyer, W.T., 1982, Comput. Biol. Med., $12179-182$.

Devine, B., 1974, Drug Intell. Clin. Pharm., 8, 650-655.

Ellis, E.F., Koysooko, R. and Levy, G., 1976, Pediatrics, 58, 542-547.

Giacoia, G., Jusko, W.J., Menke, J. and Koup, J.R., 1976, J. Pediatr., 89, 829-832.

Hendeles, L., Weinberger, M. and Bighley, L., 1978, Am. Rev. Resp. Dis., 118, 97-103.

Howard, B., 1980, Growth and Developement, in Manual of Pediatric Therapeutics, Graef, J.W. and Cone, T.E. (Eds.), Little, Brown and Co., Boston, p. 154.

Hull, J.H. and Sarubbi, F.A., 1976, Ann. Intern. Med., 85, 183-189.

Jelliffe, R.W., 1973, Ann. Intern. Med., 79, 604-605.

King, W. and Kaul, A.F., 1980, Drug Intell. Clin. Pharm., 14, 686-693.

Loughnan, P.M., Sitar, D.S., Ogilvie, R.I., Eisen, A., Fox, Z. and Neims, A.H., 1976, J. Pediatr., 88, $874-879$.

Ludden, T.M., Allen, J.P., Valtusky, W.A., Vicuna, A.V., Nappi, J.M., Hoffman, S.F., Wallace, J.E., Lalka, D. and McNay J.L., 1977, Clin. Pharmacol. Ther., 21, 287-293.

Manion, C.V., 1980, Am. J. Hosp. Pharm. 37, 225-231.

Ng, P.K., 1980, Am. J. Hosp. Pharm., 37, 225-231.

Powell, J.R. and Jackson, J.E., 1980, Counterpoint Discussion: Theophylline, in Applied Pharmacokinetics, Evans, W.E., Schentag, J.J. and Jusko, W.J. (Eds.), Applied Therapeutics Inc. San Francisco, p. 160.

Sawchuk, R.J. and Zaske, D.E., 1976, J. Pharmacokin, Biopharm., 4, 183-195.

Schentag, J.J., 1980, Aminoglycosides, in Applied Pharmacokinetics, Evans, W.E., Schentag, J.J. and Jusko, W.J. (Eds.), Applied Therapeutics Inc. San Francisco, p. 187.

Williams, D.B. and Karl, R.C., 1979, Am. J. Surg., 137, 572-576.

Winter, M.E., 1980, Basic Clinical Pharmacokinetics, Applied Therapeutics Inc., San Francisco. 